\title{
LA PRODUCCION DE LA UNIVERSIDAD ESPAÑOLA EN QUIMICA REFLEJADA EN PUBLICACIONES ESPAÑOLAS Y EXTRANJERAS
}

\author{
J. R. Pérez Alvarez-Ossorio; I. Gómez Caridad; M. J. Martín Sempere; \\ C. Urdín Caminos; C. Galbán Ferrús; S. Hernando Tundidor e I. Aguillo Caño*
}

Resumen: Se analiza la producción de la Universidad española en el campo de la Química, en el período 1990-91, utilizando Chemical Abstracts como base de datos internacional e ICYT como base de datos nacional, que recoge exhaustivamente todo lo publicado en revistas españolas. La comparación entre ambas series de datos permite deducir tendencias de publicación en el extranjero, así como datos sobre la difusión internacional de la literatura publicada en España.

Palabras clave: Química, producción científica, publicaciones científicas, Universidad, España.

\begin{abstract}
The scientific production of Spanish Universities in the field of Chemistry is analyzed for the period 1990-91. Data are obtained from Chemical Abstracts, as international database, and from ICYT as national database, which is comprehensive for articles published in Spanish journals. Comparison between both series of data permits to draw conclusions on trends to publish abroad, as well as on the international diffusion of the literature published in Spain.
\end{abstract}

Keywords: Chemistry, scientific output, scientific publications, University, Spain.

\section{Introducción}

El presente trabajo continúa la línea iniciada en un trabajo anterior (1), en el que se analizaba la producción de la Universidad española en el área de la Física, a través de la presencia de publicaciones españolas en una base de datos internacional (INSPEC), analizando de igual modo los datos recogidos en una base de datos española (ICYT), y procediendo finalmente a una comparación entre ambas series de datos.

Para realizar un estudio similar en el área de la Química se utiliza Chemical Abstracts como base de datos internacional, y de nuevo ICYT, en sus apartados de Química, como base de datos nacional. El período sobre el que se realiza el estudio es el bienio 1990-1991.

El trabajo consta, pues, de tres partes. En la primera se analiza la presencia de artículos españoles en Chemical Abstracts, tanto los publicados en revistas espanolas como los publicados en revistas extranjeras, pero únicamente los realizados en España, es decir, sin incluir los trabajos realizados por autores españoles en instituciones extranjeras. En la segunda, se realiza un análisis similar en la base de datos ICYT. Por último, en la tercera parte se comparan los datos obtenidos en

* Centro de Información y Documentación Cientifica. CSIC.

Recibido: 18-11-93. 
las dos partes precedentes, lo que permitirá estudiar, por un lado, las tendencias de publicación en el extranjero y, por otro, la difusión internacional de las publicaciones españolas estimando que un índice de dicha difusión es la proporción de trabajos publicados en revistas españolas que recoge la base de datos internacional.

\section{Análisis de los datos procedentes de Chemical Abstracts}

\subsection{Metodología}

La base de datos CA, preparada por el Chemical Abstracts Service, y cuyo contenido corresponde al repertorio impreso del mismo título, es bien conocida por los químicos de todo el mundo y se considera como una de las más exhaustivas que existen en cualquier disciplina. Se prepara a partir de unas 9.000 revistas de todo el mundo, patentes de 26 países, libros, monografias, etc. En la actualidad, contiene por encima de 10,5 millones de referencias y se actualiza cada dos semanas, incorporando de 15 a 20.000 nuevas referencias.

Las referencias de los trabajos realizados en España se obtuvieron por teledescarga, recuperando los documentos donde figura «Spain» como lugar de trabajo y cuyo año de publicación es 1990 ó 1991 . Aunque, a diferencia de INSPEC, CA incluye los nombres de todos los autores, figura, sin embargo, una sola institución, lo que conduce en la práctica a las mismas limitaciones señaladas en el trabajo anterior para INSPEC, a saber:

a) Se pierden los trabajos donde figuran autores españoles, pero donde la institución que aparece es extranjera. La práctica totalidad de estos casos se refiere a trabajos realizados por autores españoles fuera de España, que no contemplamos aquí, por lo que el error derivado de esta causa es virtualmente inexistente.

b) Los trabajos realizados en colaboración entre varias instituciones españolas se imputan siempre a la única que aparece en CA como institución principal que es, generalmente, la del primer firmante o la del autor con quien debe mantenerse la correspondencia.

c) Cuando se trata de colaboraciones' entre varios centros universitarios, los trabajos se imputan igualmente al único que figura como institución principal.

Las referencias obtenidas se transfirieron, mediante un programa desarrollado en el CINDOC (PROEL), a un formato aceptado por dBASE IV. La base de datos así creada fue la finalmente utilizada en el análisis posterior. A tal efecto, el campo «institución» fue homologado con ayuda de programas propios, desarrollados específicamente, completando y corrigiendo los resultados en forma manual, con ayuda del "Directorio de Recursos Humanos en Investigación y Desarrollo» de la Dirección General de Política Científica.

\subsection{Resultados}

Se obtuvieron de CA un total de 12.709 documentos españoles, en su gran mayoría artículos de revistas: $11.996(94 \%)$. Junto a ellos, comunicaciones a con- 
gresos, patentes y libros e informes (Figura 1). La participación relativa de la Universidad en el conjunto de trabajos es del $67,6 \%$, y se mantiene estable a lo largo de los dos años objeto del estudio (Figura 2).

\section{Figura 1}

\section{Documentos espanoles en Chemical Abstracts, anos 1990-1991}

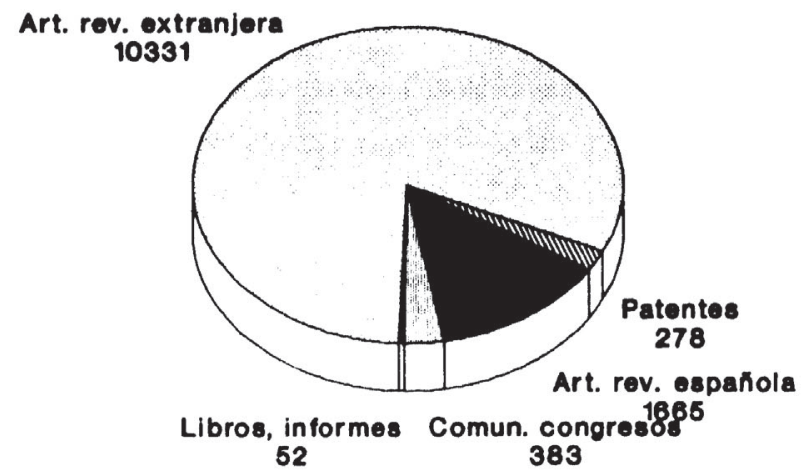

Figura 2

\section{Participación relativa de la Universidad en el Chemical Abstracts}

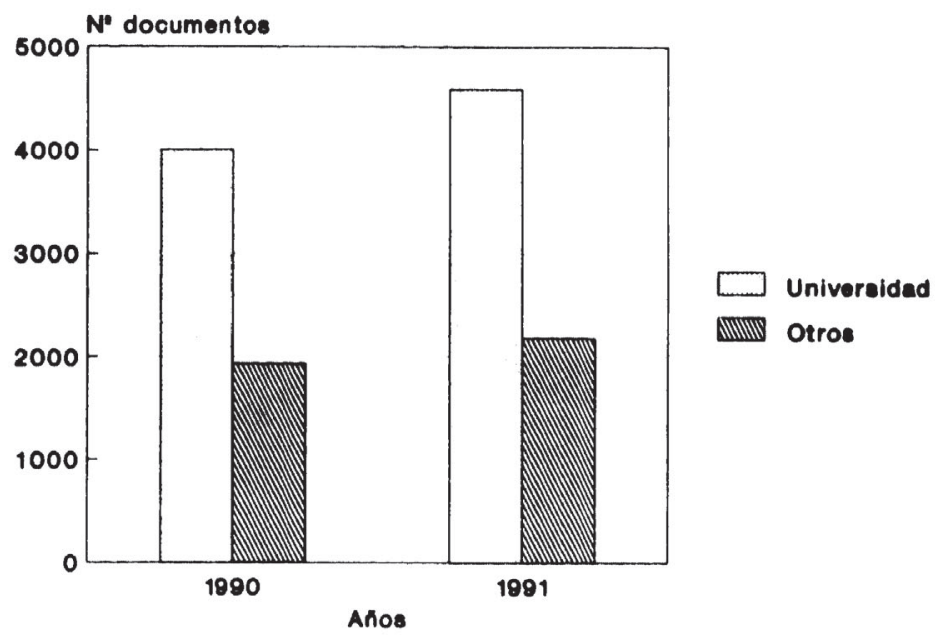

Teniendo en cuenta esta gran preponderancia de los artículos de revista y como, por otra parte, la comparación con la base de datos ICYT sólo será posible en este tipo de documentos, limitaremos en lo que sigue nuestro análisis a los artículos de revistas, ya sean éstas españolas o extranjeras. La Tabla I recoge la distribución del total de artículos de revistas, subdividiendo ya el epígrafe "otros»:

A partir de aquí, nos referiremos en exclusiva a los artículos en que ha participado la Universidad. En primer término, se ha procedido a la distribución por tipos de centros (Tabla II).

Ante todo, hay que llamar la atención sobre un hecho, ya apuntado en el trabajo anterior sobre Física, y que ahora se refuerza al referirnos a una fecha posterior, a saber, la progresiva pérdida de significación de la unidad "facultad", apareciendo los trabajos adscritos a «departamentos» que, en bastantes casos, son interfacultativos. Ello hace que no haya sido posible distribuir todos los trabajos 
procedentes de facultades de Ciencias no subdivididas entre las correspondientes secciones (físicas, químicas, matemáticas, biológicas y geológicas), permaneciendo una cantidad importante de artículos atribuidos a «facultades de Ciencias».

Tabla I

Total articulos de revistas (CA)

\begin{tabular}{lrrrr}
\hline & Rev. esp. & Rev. extr. & Total & $\%$ \\
\cline { 2 - 5 } Universidad & 977 & 7.376 & 8.353 & 70 \\
CSIC & 294 & 2.076 & 2.370 & 20 \\
Otros centros & 75 & 340 & 415 & 3 \\
Hospitales & 52 & 364 & 416 & 3 \\
Empresas & 179 & 125 & 304 & 3 \\
Sin especificar & 88 & 50 & 138 & 1 \\
$\quad$ TOTAL & 1.665 & 10.331 & 11.996 & 100 \\
\hline
\end{tabular}

Tabla II

Artículos de revistas por tipos de centros (CA)

\begin{tabular}{lrrr}
\hline & 1990 & 1991 & Total \\
\cline { 2 - 4 } Fac. Químicas & 1.287 & 1.312 & 2.599 \\
Fac. Físicas & 340 & 455 & 795 \\
Fac. Farmacia & 426 & 497 & 923 \\
Fac. Biológicas & 248 & 346 & 594 \\
Fac. Geológicas & 22 & 43 & 65 \\
Fac. Matemáticas & 7 & 3 & 10 \\
Fac. Medicina & 304 & 458 & 762 \\
Fac. Veterinaria & 98 & 143 & 241 \\
Fac. Ciencias & 626 & 663 & 1.289 \\
Otras facultades & 4 & 20 & 24 \\
$\quad$ Total facultades & 3.362 & 3.940 & 7.302 \\
& & & \\
ETSI Industriales & 134 & 185 & 319 \\
ETSI Agrónomos & 47 & 45 & 92 \\
ETSI Telecomunicación & 28 & 15 & 43 \\
ETSI Minas & 22 & 11 & 33 \\
ETSI Caminos & 8 & 22 & 30 \\
Fac. Informática & 3 & 15 & 18 \\
Otras ETS & 11 & 26 & 37 \\
$\quad$ Total ETS & 253 & 319 & 572 \\
Colegios universitarios & & & \\
Escuelas universitarias & 43 & 16 & 59 \\
Institutos universitarios & 65 & 34 & 99 \\
Sin especificar & 91 & 127 & 218 \\
$\quad$ TOTAL GENERAL & 59 & 44 & 103 \\
\hline
\end{tabular}

El conjunto de facultades representa el $88 \%$ del total, mientras que la contribución de las Escuelas Técnicas Superiores es sólo del 7\%. Por otra parte, si no se tienen en cuenta las facultades de Ciencias no subdivididas, las facultades de Química suponen el 43\% del total de facultades. Además de las facultades de Física (cuyo papel frente a las de Química es recíproco), aparecen contribuciones impor- 
tantes de las facultades de Farmacia, Medicina y Biológicas. Puede advertirse también que, aunque la contribución de las Escuelas Técnicas es pequeña, el crecimiento experimentado de 1990 a 1991 es sensiblemente superior al de las facultades (26 frente al $17 \%$ de aumento).

La distribución por universidades arroja los resultados que aparecen en la Tabla III.

Tabla III

Artículos de revistas por universidades (CA)

\begin{tabular}{|c|c|c|c|}
\hline & 1990 & 1991 & Total \\
\hline Barcelona & 420 & 464 & 884 \\
\hline Complutense & 420 & 433 & 853 \\
\hline Valencia & 246 & 314 & 560 \\
\hline Autónoma Madrid & 221 & 287 & 508 \\
\hline Santiago de Compostela & 190 & 264 & 454 \\
\hline País Vasco & 200 & 215 & 415 \\
\hline Autónoma Barcelona & 193 & 200 & 393 \\
\hline Granada & 170 & 195 & 365 \\
\hline Oviedo & 162 & 185 & 347 \\
\hline Sevilla & 146 & 185 & 331 \\
\hline Córdoba & 139 & 149 & 288 \\
\hline Valladolid & 123 & 150 & 273 \\
\hline Murcia & 126 & 145 & 271 \\
\hline Zaragoza & 142 & 121 & 263 \\
\hline Salamanca & 112 & 131 & 243 \\
\hline Málaga & 85 & 96 & 181 \\
\hline Extremadura & 83 & 92 & 175 \\
\hline La Laguna & 66 & 108 & 174 \\
\hline Politécnica Madrid & 83 & 90 & 173 \\
\hline Politécnica Cataluña & 83 & 86 & 169 \\
\hline Alcalá de Henares & 88 & 64 & 152 \\
\hline Alicante & 55 & 83 & 138 \\
\hline Baleares & 63 & 67 & 130 \\
\hline Politécnica Valencia & 38 & 62 & 100 \\
\hline León & 39 & 58 & 97 \\
\hline Politécnica Canarias & 34 & 59 & 93 \\
\hline Cantabria & 35 & 56 & 91 \\
\hline Cádiz & 35 & 28 & 63 \\
\hline Navarra & 26 & 22 & 48 \\
\hline UNED & 23 & 23 & 46 \\
\hline Castilla-La Mancha & 14 & 30 & 44 \\
\hline Vigo & - & 7 & 7 \\
\hline La Coruña & - & 4 & 4 \\
\hline Navarra Pública & - & 3 & 3 \\
\hline Sin especificar & 13 & 4 & 17 \\
\hline TOTAL & 3.873 & 4.480 & 8.353 \\
\hline
\end{tabular}

Como podría esperarse, según lo dicho anteriormente, las Universidades Politécnicas aparecen en lugares bastante bajos de la clasificación. Por otra parte, las universidades de Madrid y Barcelona (incluidas Alcalá de Henares y la UNED) concentran algo más de la tercera parte del total $(38 \%)$. 
En cuanto a las revistas más utilizadas, el recuento se ha realizado sobre el total de los 11.996 artículos (es decir, no sólo sobre los de la Universidad). Dicho total se reparte entre 1.442 revistas. Si se seleccionan las que contienen 20 o más artículos (es decir, una media de 10 por año), resultan 144 revistas, cuya distribución por países aparece en la Tabla IV.

Tabla IV

Revistas más utilizadas por paises (CA)

\begin{tabular}{lrr}
\hline & Núm. rev. & Núm. art. \\
\cline { 2 - 3 } EE. UU. & 50 & 2.190 \\
España & 24 & 1.431 \\
Holanda & 27 & 1.225 \\
Gran Bretaña & 21 & 844 \\
Alemania & 8 & 281 \\
Suiza & 3 & 159 \\
Dinamarca & 2 & 83 \\
Irlanda & 2 & 53 \\
Ucrania & 1 & 44 \\
Japón & 1 & 41 \\
Australia & 1 & 37 \\
Francia & 1 & 27 \\
Canadá & 1 & 26 \\
Portugal & 1 & 26 \\
Italia & 1 & 24 \\
$\quad$ TOTAL & 144 & 6.491 \\
\hline
\end{tabular}

Estas 144 revistas, que suponen el $10 \%$ del total, concentran el $54 \%$ del total de artículos. Tras los cuatro primeros países (la presencia de Holanda se explica porque cierto número de revistas internacionales importantes se editan en ese país) se produce una fuerte disminución, tanto en el número de revistas como en el de artículos. Por otra parte, en las revistas españolas se produce una concentración mucho mayor que en las extranjeras y así, las 24 revistas españolas que figuran en la tabla concentran el $86 \%$ del total de artículos publicados en revistas de nuestro país, mientras que las 120 revistas extranjeras concentran sólo el $49 \%$ de los artículos que se publican en el extranjero.

\subsection{Comparación con períodos anteriores}

La bibliografia sobre la presencia de trabajos españoles en Chemical Abstracts es relativamente abundante. Disponemos de datos que permiten seguir su evolución por períodos de cinco años, si bien no son datos estrictamente comparables pues, mientras los de 1975 y 1980 (2 y 3) se refieren solamente a un semestre (un volumen de Chemical Abstracts), los de 1985 (4) y los obtenidos en este trabajo para 1990 se refieren al año completo. Pero sí se pueden comparar porcentajes, y ello se resume en la Tabla V. 
Tabla V

Comparación con períodos anteriores (CA)

\begin{tabular}{lcccc}
\hline & 1975 & 1980 & 1985 & 1990 \\
\cline { 2 - 5 } & 0,49 & 0,60 & 1,00 & 1,21 \\
Trabajos esp. en CA (\%) & 50 & 58 & 67 & 69 \\
Univ. en total tr. esp. (\%) & 31 & 49 & 70 & 85 \\
Art. esp. en rev. extr. sobre total art. esp. (\%) & & \\
\hline
\end{tabular}

Como vemos, el porcentaje de trabajos españoles en Chemical Abstracts ha crecido notablemente, duplicándose con creces en un período de quince años. Este aumento corresponde en gran medida a la Universidad, cuya participación relativa en el total de trabajos españoles no ha cesado de crecer, aunque en menor grado al final del periodo. Dentro del total correspondiente a la Universidad, la participación relativa del conjunto de facultades alcanza su máximo $(96 \%)$ en 1980 para decrecer después $(94 \%$ en 1985 y $89 \%$ en 1990). Ello se debe no tanto a la participación relativa de las Escuelas Técnicas Superiores, que se mantiene prácticamente invariable, cuanto a la aparición de las Escuelas, Colegios e Institutos universitarios, prácticamente inexistentes en 1975 y 1980. Por último, resulta espectacular el aumento de la proporción de trabajos publicados en revistas extranjeras, que pasa del 31 al $85 \%$ en el período y ello en una base de datos como Chemical Abstracts, cuya cobertura de revistas españolas puede considerarse más que aceptable. Volveremos sobre este punto en la tercera parte del trabajo.

\section{Análisis de los datos procedentes de la base de datos ICYT}

\subsection{Metodología}

La base de datos ICYT, creada en 1979 por el Instituto de Información y Documentación en Ciencia y Tecnología, recoge las referencias bibliográficas de los trabajos aparecidos en unas 450 revistas españolas, en todos los campos de la Ciencia y la Tecnología. Se actualiza a un ritmo aproximado de 6.000 referencias por año.

La selección de artículos en la base de datos ICYT, con vistas a su comparación posterior con los datos procedentes de Chemical Abstracts presenta algunos problemas de difícil solución, que es preciso tener en cuenta a la hora de sacar conclusiones. El objetivo ha sido tratar de reproducir la cobertura temática de Chemical Abstracts pero mientras la clasificación de ICYT se basa en los códigos UNESCO, la clasificación de CA es específica de dicha base de datos. Por ello, se han recuperado de la base de datos ICYT todos los trabajos correspondientes a códigos UNESCO que pueden considerarse comprendidos en los subapartados de la clasificación de CA. De este modo, la muestra obtenida de ICYT es bastante exhaustiva pero, al recuperarse los apartados UNESCO completos, pueden obtenerse trabajos cuya adscripción a la Química resulta cuando menos dudosa. Por otra parte, se dará también el caso de trabajos recogidos en CA que no aparezcan en ICYT, por estar clasificados en otras rúbricas. No obstante, en el cómputo 
global y según un muestreo realizado, el número de artículos que se hallan en estos casos es reducido y el error en que se incurre es, en todo caso, ciertamente pequeño.

A diferencia de Chemical Abstracts, la base de datos ICYT recoge todas las instituciones participantes en cada trabajo, por lo que la asignación a las mismas se ha hecho utilizando las correspondientes fracciones. Hecha esta asignación, se suprime la pequeña fracción (menos del $2 \%$ ) que corresponde a centros extranjeros. La homologación y codificación de las instituciones se realizan durante la elaboración de la base de datos. Los programas de volcado y transferencia de ficheros a dBASE, asi como los que permiten segregar instituciones y asignar fracciones, han sido especialmente elaborados en el CINDOC.

\subsection{Resultados}

El número total de documentos en la base de datos ICYT, correspondiente a los apartados seleccionados y al periodo $1990-91$ es de 3.631 y su distribución entre la Universidad y las restantes instituciones se representa en la Figura 3.

Figura 3

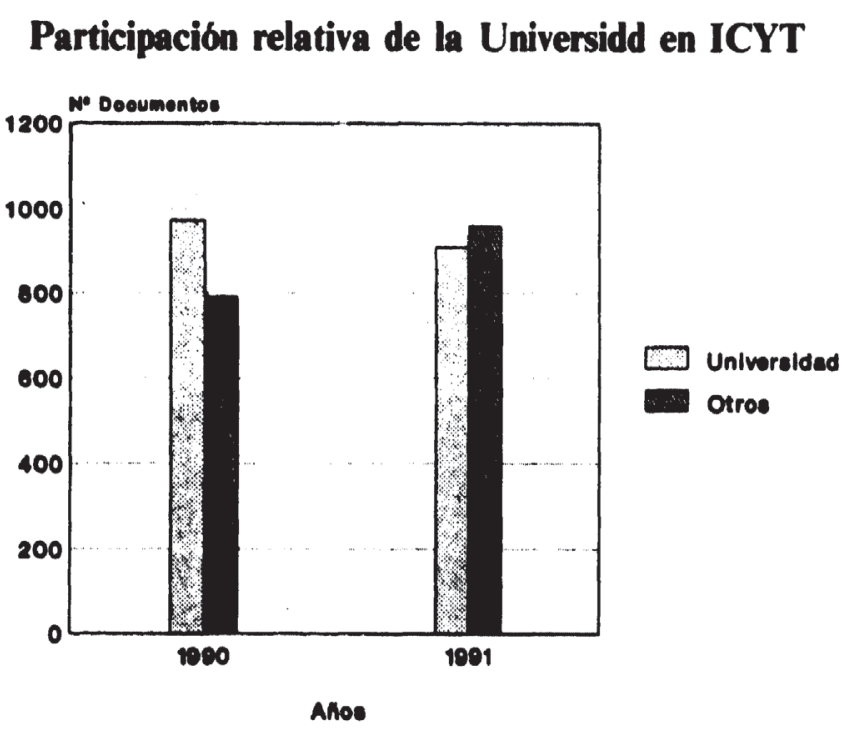

La participación relativa de la Universidad desciende de 1990 a 1991, lo que está de acuerdo con el aumento de la tendencia a publicar en el extranjero, ya apuntado y sobre el cual volveremos en la tercera parte de este trabajo. Los correspondientes datos numéricos se recogen en la Tabla VI, en la que se ha desglosado ya el epígrafe "otros".

A continuación, y siguiendo la misma sistemática que en la primera parte, se ha procedido a la distribución por tipos de centros, con los resultados que aparecen en la Tabla VII. La participación relativa del conjunto de facultades en el total baja apreciablemente frente a lo observado en Chemical Abstracts: $74 \%$ frente a $88 \%$ en CA. Por el contrario, la de las ETS se duplica con creces: $18 \%$ frente al $7 \%$ en CA. En la misma línea, la aportación de las facultades baja de 1990 a 1991, mientras la de las ETS se mantiene. Se puede, pues, decir que los artículos de índole 
Tabla VI

Total artículos (ICYT)

\begin{tabular}{lrr}
\hline & Núm. art. & $\%$ \\
\cline { 2 - 3 } Universidad & 1.882 & 52 \\
CSIC & 468 & 13 \\
Otros centros & 530 & 15 \\
Hospitales & 236 & 6 \\
Empresas & 485 & 13 \\
Sin especificar & 30 & 1 \\
$\quad$ & 3.631 & 100 \\
\hline
\end{tabular}

Tabla VII

Distribución por tipos de centros (ICYT)

\begin{tabular}{lrrr}
\hline & 1990 & 1991 & Total \\
\cline { 2 - 4 } Fac. Químicas & 293 & 289 & 582 \\
Fac. Físicas & 33 & 40 & 73 \\
Fac. Farmacia & 178 & 143 & 321 \\
Fac. Biológicas & 58 & 88 & 146 \\
Fac. Geológicas & 28 & 38 & 66 \\
Fac. Matemáticas & 1 & - & 1 \\
Fac. Medicina & 56 & 27 & 83 \\
Fac. Veterinaria & 57 & 26 & 83 \\
Fac. Ciencias & 18 & 3 & 21 \\
Otras facultades & 13 & 5 & 18 \\
$\quad$ Total facultades & 735 & 659 & 1.394 \\
& & & \\
ETSI Industriales & 71 & 78 & 149 \\
ETSI Agrónomos & 35 & 36 & 71 \\
ETSI Telecomunicación & 8 & 2 & 10 \\
ETSI Minas & 25 & 18 & 43 \\
ETSI Caminos & 14 & 20 & 34 \\
Fac. Informática & 2 & - & 2 \\
Otras ETS & 12 & 16 & 28 \\
$\quad$ Total ETS & 167 & 170 & 337 \\
& & & \\
Colegios universitarios & 17 & 9 & 26 \\
Escuelas universitarias & 36 & 44 & 80 \\
Institutos universitarios & 18 & 27 & 45 \\
$\quad$ TOTAL GENERAL & 973 & 909 & 1.882 \\
\hline
\end{tabular}

más tecnológica, procedentes de las Escuelas Técnicas, tienen una mayor presencia relativa en la base de datos ICYT o, lo que es lo mismo, que se publican en buena medida en revistas de carácter más local, no recogidas por Chemical Abstracts. Por último, cabe subrayar la importante contribución de las facultades de Farmacia.

La correspondiente distribución por universidades aparece en la Tabla VIII. En comparación con CA (Tabla III) y como era de esperar según lo dicho ante- 
riormente, se observa un fuerte ascenso de las Universidades Politécnicas; por otra parte, se produce una fuerte bajada de las Universidades Autónomas de Madrid y Barcelona que, según veremos después, ofrecen una fuerte tendencia a publicar en el extranjero, como ya ocurriera también en Física. Finalmente, la concentración en las universidades de Madrid y Barcelona (incluidas Alcalá de Henares y la UNED) es la misma que en CA: $38 \%$.

Tabla VIII

Distribución por universidades (ICYT)

\begin{tabular}{|c|c|c|c|}
\hline & 1990 & 1991 & Total \\
\hline Complutense & 98 & 103 & 201 \\
\hline Barcelona & 74 & 95 & 169 \\
\hline Santiago de Compostela & 64 & 78 & 142 \\
\hline Politécnica Cataluña & 59 & 66 & 125 \\
\hline Politécnica Madrid & 53 & 58 & 111 \\
\hline Valencia & 42 & 44 & 86 \\
\hline Granada & 62 & 23 & 85 \\
\hline Sevilla & 40 & 39 & 79 \\
\hline Oviedo & 40 & 36 & 76 \\
\hline La Laguna & 32 & 43 & 75 \\
\hline País Vasco & 35 & 39 & 74 \\
\hline Murcia & 32 & 25 & 57 \\
\hline Córdoba & 28 & 26 & 54 \\
\hline Politécnica Valencia & 25 & 29 & 54 \\
\hline Zaragoza & 37 & 16 & 53 \\
\hline Extremadura & 38 & 13 & 51 \\
\hline Valladolid & 26 & 22 & 48 \\
\hline Salamanca & 23 & 24 & 47 \\
\hline Autónoma Barcelona & 19 & 22 & 41 \\
\hline Navarra & 24 & 14 & 38 \\
\hline Málaga & 21 & 16 & 37 \\
\hline Autónoma Madrid & 23 & 13 & 36 \\
\hline Alcalá de Henares & 17 & 10 & 27 \\
\hline Cantabria & 10 & 13 & 23 \\
\hline Baleares & 9 & 6 & 15 \\
\hline Castilla-La Mancha & 6 & 9 & 15 \\
\hline UNED & 8 & 6 & 14 \\
\hline Cádiz & 6 & 7 & 13 \\
\hline Politécnica Canarias & 9 & 2 & 11 \\
\hline Alicante & 7 & 3 & 10 \\
\hline León & 6 & 4 & 10 \\
\hline Vigo & - & 3 & 3 \\
\hline Navarra Pública & - & 2 & 2 \\
\hline TOTAL & 973 & 909 & 1.882 \\
\hline
\end{tabular}

\section{Comparación de los datos obtenidos en las bases de datos CA e ICYT}

En el apartado anterior, hemos apuntado ya algunos datos derivados de la comparación entre los resultados obtenidos en las bases de datos CA e ICYT. En esta tercera parte del trabajo, nos proponemos dos objetivos: en primer término, 
analizar las tendencias de publicación en España y en el extranjero y, en segundo lugar, obtener datos sobre la difusión internacional de los trabajos publicados en revistas españolas. Es claro que la comparación entre los datos procedentes de CA e ICYT presenta una serie de dificultades y limitaciones, derivadas de los distintos criterios de selección y de clasificación, y también de lo ya advertido sobre la distinta forma de asignación de los trabajos entre las distintas instituciones participantes en los mismos: asignación a una sola institución en CA y por fracciones en ICYT. Pese a ello, estimamos que los resultados que se obtienen en esta comparación son, cuando menos, indicativos y constituyen una primera aproximación susceptible de una profundización ulterior.

\subsection{Tendencias a publicar en el extranjero}

En el análisis que sigue, se toman como trabajos publicados en España los que se recogen en la base de datos ICYT, que se considera exhaustiva a este respecto; y, como trabajos publicados en el extranjero, los recogidos por Chemical Abstracts, publicados en revistas no españolas. Los resultados se representan en la Figura 4, y los datos numéricos se recogen en la Tabla IX, donde el epígrafe "otros» figura ya desglosado.

Figura 4

Documentos españoles en ICYT y CA, años 1990-1991

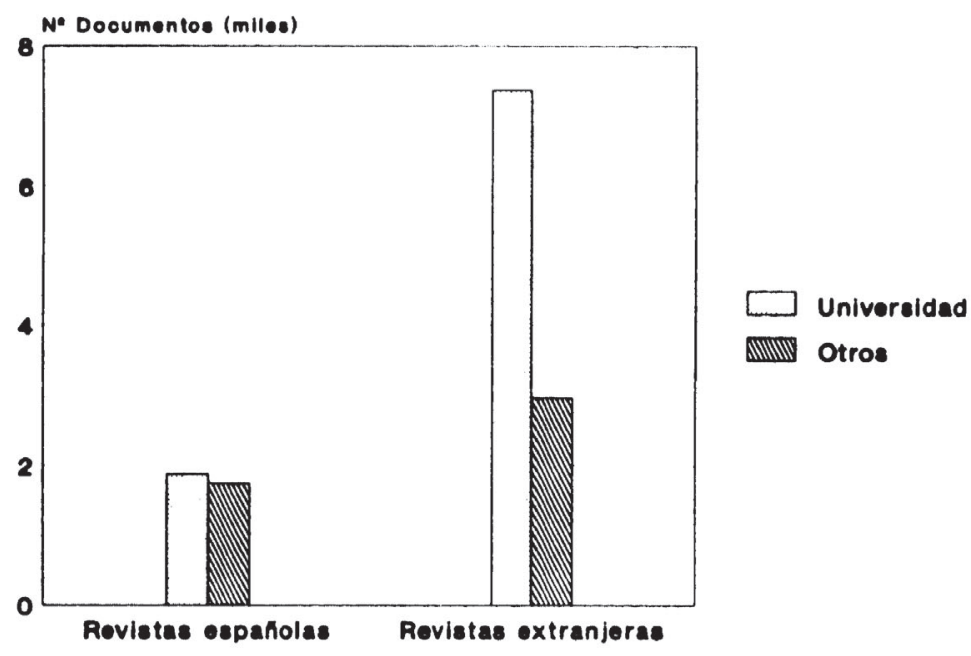

Como puede verse, la tendencia a publicar en el extranjero es sensiblemente más alta en la Universidad. En cuanto a los otros sectores, la cifra del CSIC es del mismo orden que la de la Universidad, mientras baja sensiblemente para los demás, especialmente en lo que concierne a las empresas, como por otra parte podria preverse. De 1990 a 1991, la tasa sube en la Universidad (del 77 al $81 \%$ ), y baja ligeramente en el conjunto de los otros sectores (64 al 62\%), manteniéndose en el CSIC.

A continuación, y refiriéndonos ya exclusivamente a los trabajos procedentes de la Universidad, hemos realizado la distribución por tipos de centros, con los resultados que aparecen en la Tabla X. Sobre esta tabla cabe hacer las siguientes consideraciones: en primer término, la tendencia a publicar en el extranjero es 
notablemente superior en el conjunto de facultades que en las Escuelas Técnicas Superiores. En lo que atañe a los otros centros universitarios, el comportamiento de los institutos universitarios es análogo al de las facultades, mientras que la tendencia a publicar en el extranjero baja bastante en los colegios y, sobre todo, en las escuelas universitarias, la mayoría de las cuales son también escuelas técnicas.

Tabla IX

Tendencia a publicar en el extranjero

\begin{tabular}{lcccc}
\hline & Rev. esp. & Rev. extr. & Total & o \\
\cline { 2 - 5 } Universidr. \\
\cline { 2 - 5 } Otros & 1.882 & 7.376 & 9.258 & 80 \\
CSIC & 1.749 & 2.955 & 4.704 & 63 \\
Otros centros & 468 & 2.076 & 2.544 & 82 \\
Hospitales & 530 & 340 & 870 & 39 \\
Empresas & 236 & 364 & 600 & 61 \\
Sin especificar & 485 & 125 & 610 & 20 \\
TOTAL & 30 & 50 & 80 & 62 \\
\hline
\end{tabular}

Tabla X

Artículos en revistas espanolas y extranjeras por tipos de centros

\begin{tabular}{|c|c|c|c|c|}
\hline & Rev. esp. & Rev. extr. & Total & $\%$ rev. ex. \\
\hline Fac. Químicas & 582 & 2.231 & 2.813 & 79 \\
\hline Fac. Físicas & 73 & 759 & 832 & 91 \\
\hline Fac. Farmacia & 321 & 756 & 1.077 & 70 \\
\hline Fac. Biológicas & 146 & 579 & 725 & 80 \\
\hline Fac. Geológicas & 66 & 50 & 116 & 43 \\
\hline Fac. Matemáticas & 1 & 10 & 11 & 91 \\
\hline Fac. Medicina & 83 & 721 & 804 & 90 \\
\hline Fac. Veterinaria & 83 & 207 & 290 & 71 \\
\hline Fac. Ciencias & 21 & 1.168 & 1.189 & 98 \\
\hline Otras facultades & 18 & 17 & 35 & 49 \\
\hline Total facultades & 1.394 & 6.498 & 7.892 & 82 \\
\hline ETSI Industriales & 149 & 266 & 415 & 64 \\
\hline ETSI Agrónomos & 71 & 78 & 149 & 52 \\
\hline ETSI Telecomunicaciones & 10 & 39 & 49 & 79 \\
\hline ETSI Minas & 43 & 13 & 56 & 23 \\
\hline ETSI Caminos & 34 & 25 & 59 & 42 \\
\hline Fac. Informática & 2 & 18 & 20 & 90 \\
\hline Otras ETS & 28 & 30 & 58 & 52 \\
\hline Total ETS & 337 & 469 & 806 & 58 \\
\hline Colegios universitarios & 26 & 44 & 70 & 63 \\
\hline Escuelas universitarias & 80 & 76 & 156 & 49 \\
\hline Institutos universitarios & 45 & 208 & 253 & 82 \\
\hline Sin especificar & - & 81 & 81 & 100 \\
\hline TOTAL GENERAL & 1.882 & 7.376 & 9.258 & 80 \\
\hline
\end{tabular}


En cuanto a los datos de cada tipo de facultad, hay que llamar la atención sobre la distorsión causada por las facultades de Ciencias no subdivididas: en el caso de la base de datos ICYT (revistas españolas), al constar todas las instituciones participantes en los trabajos, ha sido posible reasignar la mayoría de ellos entre las distintas secciones, lo que no ha sido posible en CA (revistas extranjeras). Sin duda, si esta reasignación hubiera sido posible, subiría apreciablemente el porcentaje de las facultades de Química.

Por último, cabe constatar que los porcentajes más bajos de publicación en el extranjero se dan en las facultades de Geológicas y en las ETS de Ingenieros de Minas y de Caminos, la índole de cuyos trabajos supone muchas veces su publicación en revistas locales.

Finalmente, la Tabla XI recoge los resultados de la distribución por universidades.

Tabla XI

Artículos en revistas espanolas y extranjeras por universidades (ICYT)

\begin{tabular}{|c|c|c|c|c|}
\hline & Rev. esp. & Rev. extr. & Total & $\%$ r. extr. \\
\hline Barcelona & 169 & 801 & 970 & 82 \\
\hline Complutense & 201 & 718 & 919 & 78 \\
\hline Valencia & 86 & 5() 4 & 590 & 85 \\
\hline Autónoma Madrid & 36 & 487 & 523 & 93 \\
\hline Santiago de Compostela & 142 & 376 & 518 & 72 \\
\hline País Vasco & 74 & 373 & 447 & 83 \\
\hline Autónoma Barcelona & 41 & 373 & 414 & 90 \\
\hline Granada & 85 & 321 & 406 & 79 \\
\hline Sevilla & 79 & 296 & 375 & 79 \\
\hline Oviedo & 76 & 292 & 368 & 79 \\
\hline Córdoba & 54 & 264 & 318 & 83 \\
\hline Zaragoza & 53 & 245 & 298 & 82 \\
\hline Murcia & 57 & 235 & 292 & 80 \\
\hline Valladolid & 48 & 244 & 292 & 83 \\
\hline Salamanca & 47 & 217 & 264 & 82 \\
\hline Politécnica Madrid & 111 & 142 & 253 & 56 \\
\hline Politécnica Cataluña & 125 & 125 & 250 & 50 \\
\hline La Laguna & 75 & 142 & 217 & 65 \\
\hline Extremadura & 51 & 140 & 191 & 73 \\
\hline Málaga & 37 & 148 & 185 & 80 \\
\hline Alcalá de Henares & 27 & 141 & 168 & 84 \\
\hline Politécnica Valencia & 54 & 92 & 146 & 6.3 \\
\hline Baleares & 15 & 122 & 137 & 89 \\
\hline Alicante & 10 & 127 & 137 & 93 \\
\hline Cantabria & 23 & 88 & 111 & 79 \\
\hline Lcón & 10 & 95 & 105 & 90 \\
\hline Politécnica Canarias & 11 & 92 & 103 & 89 \\
\hline Navarra & 38 & 32 & 70 & 46 \\
\hline Cádiz & 13 & 54 & 67 & 80 \\
\hline Castilla-La Mancha & 15 & 35 & 50 & 70 \\
\hline UNED & 14 & 32 & 46 & 69 \\
\hline Vigo & 3 & 6 & 9 & 67 \\
\hline Navarra Pública & 2 & 2 & 4 & 50 \\
\hline La Coruña & & 4 & 4 & 100 \\
\hline Sin especificar & & 11 & 11 & 100 \\
\hline TOTAL & 1.882 & 7.376 & 9.258 & 80 \\
\hline
\end{tabular}


Si limitamos el análisis a las universidades que aportan más de 200 trabajos (y que suponen en conjunto el $83 \%$ del total de artículos), veremos que los porcentajes de publicación en el extranjero se mueven en torno a la media del $80 \%$, excepto para las dos excepciones a que ya hemos tenido ocasión de referirnos: las Universidades Autónomas de Madrid y Barcelona, que destacan por sus altos porcentajes de publicación en el extranjero, y las Universidades Politécnicas que, como era de esperar por lo ya indicado anteriormente, presentan porcentajes mucho más bajos. Además, cabe citar los porcentajes relativamente bajos de Santiago y La Laguna.

\subsection{Difusión internacional de la literatura química publicada en España}

En este punto, vamos a comparar los trabajos publicados en revistas españolas, es decir, los recogidos por ICYT, con la proporción de ellos que a su vez recoge Chemical Abstracts, entendiendo que tal proporción representa una medida de la difusión internacional de lo que se publica en las revistas españolas. Los datos aparecen en la Tabla XII.

\section{Tabla XII}

Proporción de artículos de revistas espanoolas recogidos por $\mathrm{CA}$

\begin{tabular}{lccc}
\hline & En ICYT & De ellos en CA & \% en $C A$ \\
\cline { 2 - 4 } Universidad & 1.882 & 977 & 52 \\
Otros & 1.749 & 688 & 39 \\
CSIC & 468 & 294 & 63 \\
Empresas & 485 & 179 & 37 \\
Varios & 796 & 215 & 27 \\
TOTAL & 3.631 & 1.665 & 46 \\
\hline
\end{tabular}

Como se ve, el índice de difusión en la Universidad es sensiblemente superior al del conjunto de las demás instituciones. Pero si se desglosa el epígrafe "otros», el indice del CSIC es incluso superior al de la Universidad.

\section{Conclusiones}

1. La participación de la Universidad en la producción científica española en Química es muy importante. Referida a artículos de revistas, representa el $70 \%$ de los trabajos españoles recogidos por Chemical Abstracts y el $52 \%$ de los recogidos en los apartados relacionados con la Química de la base de datos ICYT.

2. Esta producción se publica mayoritariamente en revistas extranjeras. Si, como trabajos publicados en España se toman los procedentes de la base de datos ICYT, y como trabajos publicados en el extranjero los recogidos por Chemical Abstracts publicados en revistas no españolas, resulta que la tendencia a publicar 
en el extranjero es en la Universidad del $80 \%$, y desciende al $63 \%$ para «otros organismos». No obstante, la subdivisión del epígrafe "otros organismos» indica que en los centros del CSIC la tendencia a publicar en el extranjero es similar a la de la Universidad $(82 \%)$, mientras que son las restantes instituciones y, sobre todo, las empresas, las que hacen descender el porcentaje.

3. En la producción de la Universidad recogida por Chemical Abstracts, el conjunto de facultades representa el $88 \%$ del total, mientras que las Escuelas Técnicas Superiores alcanzan sólo el 7\%. Por el contrario, en la base de datos ICYT el peso relativo de las facultades baja al $74 \%$, mientras el de las Escuelas Técnicas sube hasta el $18 \%$, como resultado de una mayor presencia relativa de los centros tecnológicos en las revistas nacionales no recogidas por CA. La tendencia a publicar en el extranjero es del $82 \%$ en las facultades y del $58 \%$ en las Escuelas Técnicas Superiores.

4. Por universidades, la de Barcelona y la Complutense ocupan los dos primeros lugares en ambas bases de datos. Las diferencias más notables se refieren a las Universidades Autónomas de Madrid y Barcelona, que ocupan lugares destacados en Chemical Abstracts y descienden notablemente en la base de datos ICYT. Inversamente, las Universidades Politécnicas de Madrid y Cataluña ocupan lugares altos en ICYT y bajos en CA. Ello se corresponde con la tendencia a publicar en el extranjero, que es máxima en las Universidades Autónomas (93 y $90 \%$ ) y mínima en las Politécnicas (56 y 50\%).

5. $\mathrm{Si}$, como medida de la difusión internacional de los trabajos publicados en revistas españolas tomamos la proporción de trabajos incluidos en la base de datos ICYT que, a su vez, recoge Chemical Abstracts, vemos que dicha proporción es del $52 \%$ para los trabajos de la Universidad y del 39\% para los de otros organismos. De nuevo la subdivisión del epígrafe «otros» arroja un porcentaje incluso superior para los centros del CSIC $(63 \%)$, mientras que ya es bastante inferior para las restantes instituciones.

6. En cuanto al país de edición de las revistas más utilizadas, dato éste tomado únicamente en Chemical Abstracts, figuran a la cabeza las norteamericanas, seguidas de las españolas, holandesas (incluidas las internacionales editadas en Holanda) y británicas. La concentración de trabajos es notablemente superior en las revistas españolas que en las extranjeras.

7. Se ha podido seguir la evolución de la presencia de trabajos españoles en Chemical Abstracts, por periodos de cinco años, entre 1975 y 1990. El porcentaje de trabajos españoles en CA pasa del $0,49 \%$ en 1975 al $1,21 \%$ en 1990. La participación de la Universidad en este conjunto sube del 50 al 69\% en el periodo. Por último, si dentro de Chemical Abstracts se compara el número de trabajos en revistas españolas y extranjeras, la proporción de trabajos en estas últimas crece espectacularmente desde el $31 \%$ en 1975 al $85 \%$ en 1990.

\section{Bibliografia}

1. PEREZ ALVAREZ-OSSORIO, J. R.; (GOMEZ CARIDAD, I., y otros. La producción de la Universidad española en Física, reflejada en las publicaciones españolas y extranjeras, Rev. Esp. Doc. Cient., 14, 4, 1991, pp. 428-444. 
2. PEREZ ALVAREZ-OSSORIO, J. R. Análisis de la literatura química española a través del volumen 83 de Chemical Abstracts, Afinidad $X X X I I I, 337,1976$, pp. 435443.

3. PEREZ ALVAREZ-OSSORIO, J. R. Análisis comparativo de la literatura química española en 1975 y 1980 a través de Chemical Abstracts, Rev. Esp. Doc. Cient., 6, 1, 1983, pp. 33-48.

4. GOMEZ CARIDAD, I., y MENDEZ MIAJA, A. La producción científica de la Universidad española recopilada por las bases internacionales de datos bibliográficos en 1985. Madrid, 1986. 